\title{
Indoor Positioning: Novel Approach for Bluetooth Networks using RSSI Smoothing
}

\author{
J.A.D.C Anuradha Jayakody \\ Sri Lanka Institute of Information Technology \\ New Kandy Road, Malabe, \\ Sri Lanka \\ Dinusha Chathurangi \\ Sri Lanka Institute of Information Technology \\ New Kandy Road, Malabe, \\ Sri Lanka
}

\author{
Shashika Lokuliyana \\ Sri Lanka Institute of Information Technology \\ New Kandy Road, Malabe, \\ Sri Lanka \\ Demini Vithana \\ Sri Lanka Institute of Information Technology \\ New Kandy Road, Malabe, \\ Sri Lanka
}

\begin{abstract}
In recent years, localization and navigation have been important topics in research. The most popular navigation system is an outdoor navigation with "GPS"; however, the positioning within indoor environments is not possible with "GPS", so it is resulting in limited operation for indoor environments. In order to overcome this limitation, this paper discussed Bluetooth Low Energy technology based localization model. The "BLE" provides several major forms of parameters linked to location estimation such as "RSSI" and "LQI". In real time applications such as object tracking and distance estimates require continuous reception of RSSI measurements to estimate the position of the object accurately. Nevertheless, on that point, there are some constraints such as signal attenuation, signal loss, multipath effects, temperature, reflection, a human body and other communication signals. Hence, this research work considered the "RSSI" smoothing approaches. Although there are so many solutions, no RSSI smoothing method has been recognized as a standard method. This paper presents a Feedback filter together with shifting technique at distance domain to reduce fluctuations of the real-time signals. Experiments show that the probability of locating errorless and it is better than the other existing interference avoidance algorithms.
\end{abstract}

\section{General Terms}

Indoor navigation, Localization, i-Beacon

Keywords

LQI, BLE, RSSI, Feedback Filter, Shifting Technique.

\section{INTRODUCTION}

The growth of wireless and mobile communications technologies offers new possibilities for context driven information systems [1]. Precisely, at present mobile phones are armed with several radio-frequency technologies, like Global Positioning System (GPS), Wi-Fi and Bluetooth and so forth. Hence, most of the mobile devices have an ability to provide geographical positions with the assistance of GPS. Though it is effective to provide outdoor localization, an indoor localization is not accurate due to the poor signal quality of the GPS.The most of the researchers have been working with Bluetooth Low Energy (BLE) technology since last decades with the Received Signal Strength Indicators (RSSI).This paper presents a study of the Bluetooth signal as a source of information for the locating systems to provide a solution to find the exact indoor location. The proposed test environment consists of three beacon nodes connected network where each of them is composed of user's mobile telephone sets. It should be worked by inquiring the Received Signal Strength Indicators (RSSI) values of all the visible Bluetooth devices and may need to employ a fingerprinting technique, so it determines the current location of a person. Established in the Experimental Results of testing on the RSSI values, shifting technique, a method has been proposed, which can find the static RSSI values for the locating system. The experimental results show that the proposed method can reduce the average error.

This paper is organized as following. Section II discusses Literature Survey. Section III discusses Research Methodology. Section IV discusses results and evidence. Lastly, Section V discusses Conclusion and Future work.

\section{LITERATURE SURVEY}

The arrival of Bluetooth Low Energy (BLE) creates opportunities for great innovations. It is a mainstream technology available on the latest mobile devices. Ling Pei et al. [2], [3] present their system that finds the location using fingerprinting. According to them, the position is computed using the RSSI probability distribution combined. The accuracy obtained has a standard deviation of 10 meters. According to Fazli Subhan et al. [4] they stated that the uses trilateration for computing the status and the distance is gauged based on the radio propagation model combined with the Gradient filter for cutting down the interference. The accuracy obtained using this method is 2.67 meters. There are several recent commercial products that offer the hardware and software to help to find objects [5], [6], based on BLE. However, they all give only information regarding the intensity of the communication, or whether the object is within the range or not.

Localization error can be decreased by simultaneously observing RSSI, so the beacon nodes provide information about RSSI of a received Bluetooth signal. Thus, the sifting algorithm proposed and developed in this paper is to determine the relation between RSSI and range, which would be applied in real-time location. Lots of work has been done on RSSI smoothing [7], [8], [9] focus on optimizing correlation model between RSSI and distance, while [10] is interested in establishing the relational database for RSSI and distance. According to Lechner et al. [11] they introduce a sensor measurement model in the particle filter framework that combines a Wi-Fi signal propagation model [12] and 
fingerprinting technique for locating. In [13] the authors presented Bluetooth based indoor positioning Trilateration technique. The accuracy obtained at room level is about 4.56 meters. In [14], the author extended the idea of Kotanen [15] to Wireless Local Area Networks using Extended Kalman Filter. The average error using KNN approach is 3.37 meters and 2.11 meters using extended Kalman filter.

According to the above literature reviews which related to RSSI smoothing in the area of localization, it still needs special attention. This paper has been proposed and achieved filtered RSSI output using the shifting technique.

\section{RESEARCH METHODOLOGY RSSI Acquisition Layer}

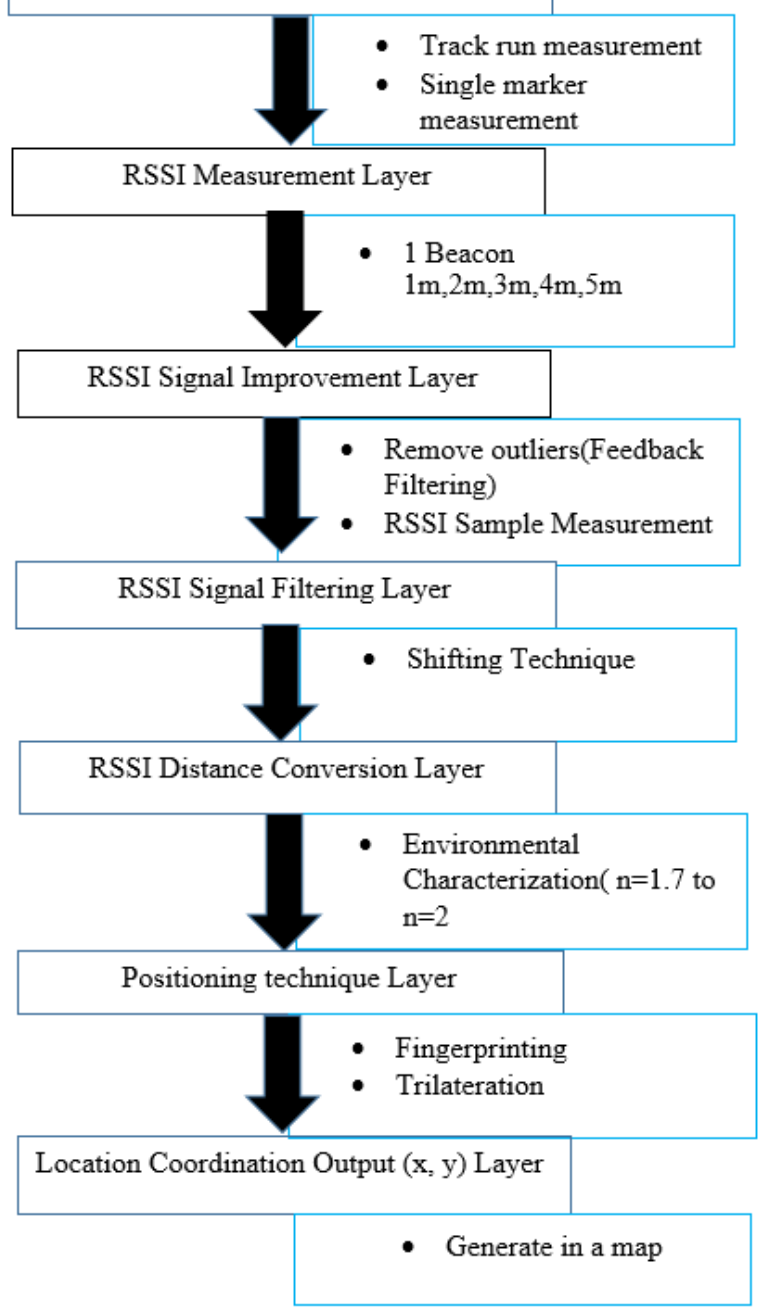

Fig 1: Block diagram of the proposed indoor positioning

This research focuses on positioning a mobile node within the beacon network using RSSI smoothing approach. In order to produce good results regarding accuracy, authors design an algorithm for indoor positioning to remove inconsistent data. The intent of this algorithm is to generate the right position by removing unwanted information. Fig. 1 depicts a block diagram which provides an overview of the proposed indoor positioning methodology.

\subsection{RSSI Acquisition}

Bluetooth RSSI readings tend to change rather significantly. The deviation between the maximum value and the minimum value is high; then it is difficult to judge the distance using
Bluetooth RSSI raw data. Hence, RSSI acquisition carried out in two stages. The track runs measurement and single marker measurement. The first entire area is split up into a rectangular grid of square blocks. In single marker measurement at each of these blocks, measure the RSSIs 20 times to predict real measurements. Although single marker measurement is done with selected locations without following a line, these approaches remain at the ranging aspect. Nevertheless, track run measurement is collecting RSSIs while moving the object. Combinations of these two methods are utilized to collect RSSI values

\subsection{RSSI Measurement}

The accuracy of the indoor positioning system is greatly dependent on the parameters selected for estimation and the measurements obtained from the environment. However, the measurements are corrupted by various environmental conditions such as temperature, reflection, presence of obstacles, the human body, and other communication signals. Thus, we need to get rid of the outliers and filter the measurements, for those proposed algorithms include "Feedback Filter."

\subsection{RSSI Signal Improvement}

The feedback filter used for eliminating noise in different environments is the filter utilized in this algorithm. If a filter approximation is employed, it can be expressed as where $\alpha$ represents the weighted value. In this equation, although the range of $\alpha$ value is $0-1$, here the variable $\alpha$ is 0.75 . RSSI $n$ has represented the most recently measured value, and RSSI $n-1$ represents the previous averaged value. This approach ensures that a large difference in RSSI values will be smoothed:

$$
R S S I_{\text {smoot } h}=\alpha * R S S I_{n}+(1-\alpha) * R S S I_{n-1} \rightarrow 1
$$

This means that the average RSSI value corresponding to the signal strength at a distance depends on both the previous averaged value and the most recently measured value.

\subsection{RSSI Signal Filtering}

Even though after getting rid of outliers, it is hard to track a RSSI range for a special position, so the system is tested using average filtering and novel shifting algorithm. Test results were verified shifting algorithm smoothen the RSSI values better than average filtering. Finally, algorithm consists of novel filtering technique (Shifting algorithm) for placing a target node using RSSI.

In this research, collect the RSSI data using track run and single marker measurement. Hence, we defined a vector to store the training data which are coming after performing feedback filtering. Fig. 2 depicts shifting technique performing continuously: while navigating and every time takes an average of 20 values. 


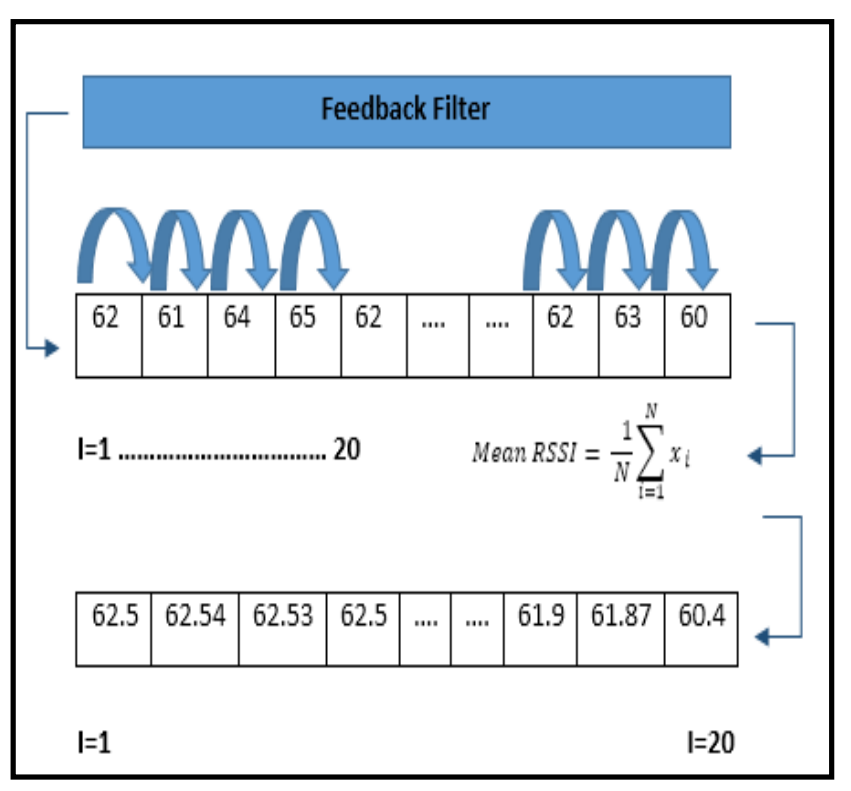

\subsection{RSSI distance conversion}

To obtain distances based on the measured RSSI the mathematical model in the equation as the following.

$$
\begin{aligned}
& R S S I=-\left(10 n \log _{10} d+A\right) \longrightarrow \\
& d=10^{\frac{A-R S S I}{10 * n}} \longrightarrow{ }^{2}
\end{aligned}
$$

Where, RSSI: is the RSSI value received (dBm), $\mathrm{n}$ : is the path-loss exponent is the distance, A: is 3 the RSSI value at a " 1 " meter reference distance.

It is observable through values obtain from beacons, for the selected area RSSI values are variable according to the $n$ value. This $n$ value recognized as the path loss exponent and is extremely dependent on the surroundings. To examine the environmental characterization drew a graph (fig. 3) Using collected information from the investigational area and compared it to when $n=1.7$ to $n=2$.

Fig 2: The novel shifts algorithm

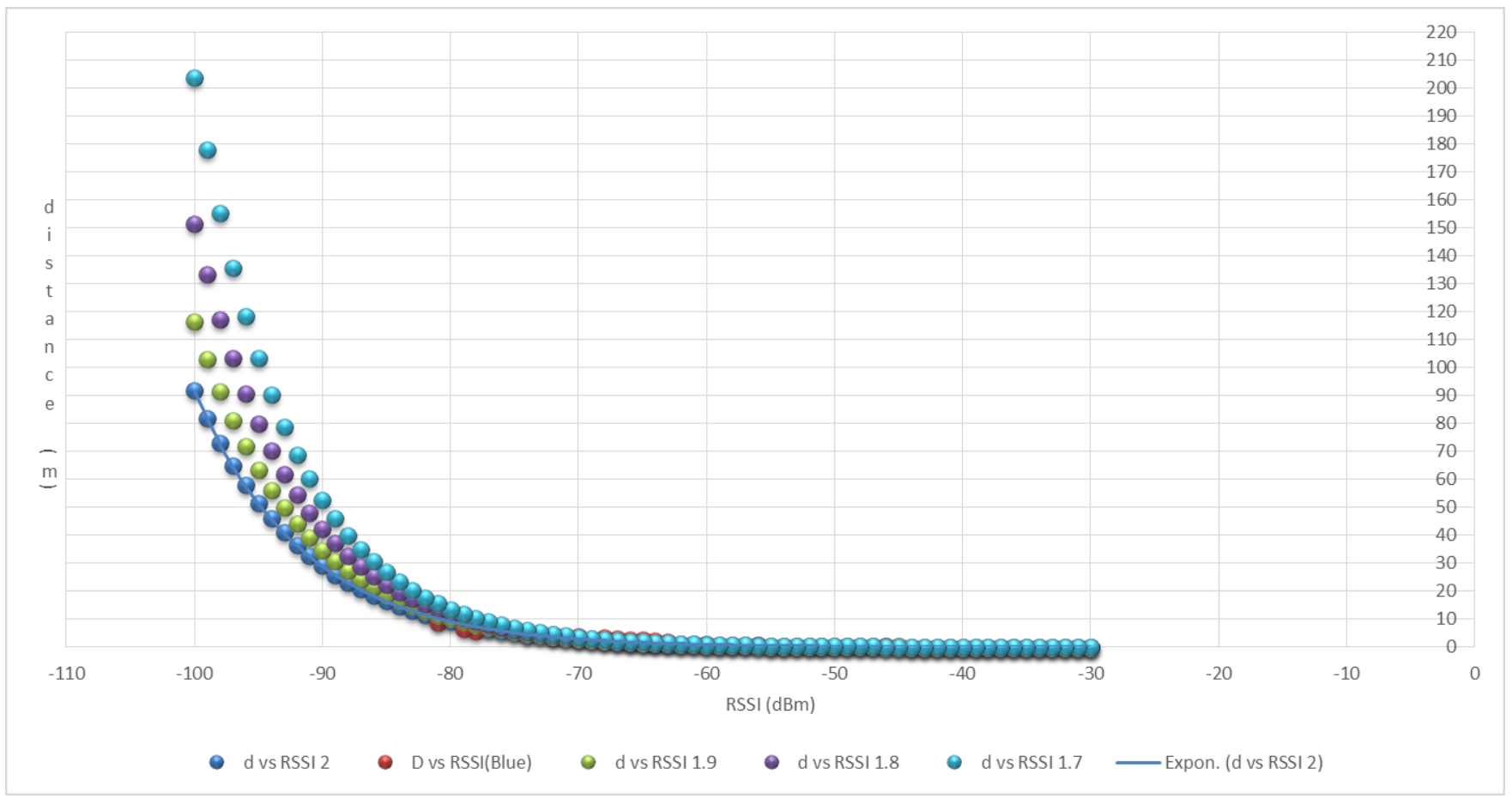

Fig 3: Environmental characterization variation

\subsection{Positioning Technique}

Trilateration algorithm will choose the estimated distance from at least three beacons as inputs. The method for localization requires the user's distance from three reference nodes with unknown coordinates because it uses the geometry of triangle [16]. Each beacon location represents a vertex a triangle while each estimated distance represents a side of the triangle, so the process is called trilateration. This test designed to take user input values from the beacon. Hence plan to identify the corresponding distance between the user and the beacons (Fig. 4) with user input distances to Beacon (A, B, C,). The equations (4), (5), (6), and (7) were employed to determine the $\mathrm{X}$ and $\mathrm{Y}$ coordinates of the user.

$$
\begin{array}{lll}
v_{a}=\left[\left(d_{b}^{2}-d_{c}^{2}\right)-\left(x_{b}^{2}-x_{c}^{2}\right)-\left(y_{b}^{2}-y_{c}^{2}\right)\right] / 2 & \rightarrow & 4 \\
v_{b}=\left[\left(d_{b}^{2}-d_{a}^{2}\right)-\left(x_{b}^{2}-x_{a}^{2}\right)-\left(y_{b}^{2}-y_{c}^{2}\right)\right] / 2 & \rightarrow & 5 \\
y=\left[v_{b}\left(x_{c}-x_{b}\right)-v_{a}\left(x_{a}-x_{b}\right)\right] /\left[\left(y_{a}-\right.\right. & & \\
\left.y_{b}\right)\left(x_{c}-x_{b}\right)-\left(y_{c}-y_{b}\right)\left(x_{a}-x_{b}\right) & \rightarrow & 6 \\
x=\left[v_{a}-y\left(y_{c}-y_{b}\right)\right] /\left[x_{c}-x_{b}\right] & \rightarrow & 7
\end{array}
$$




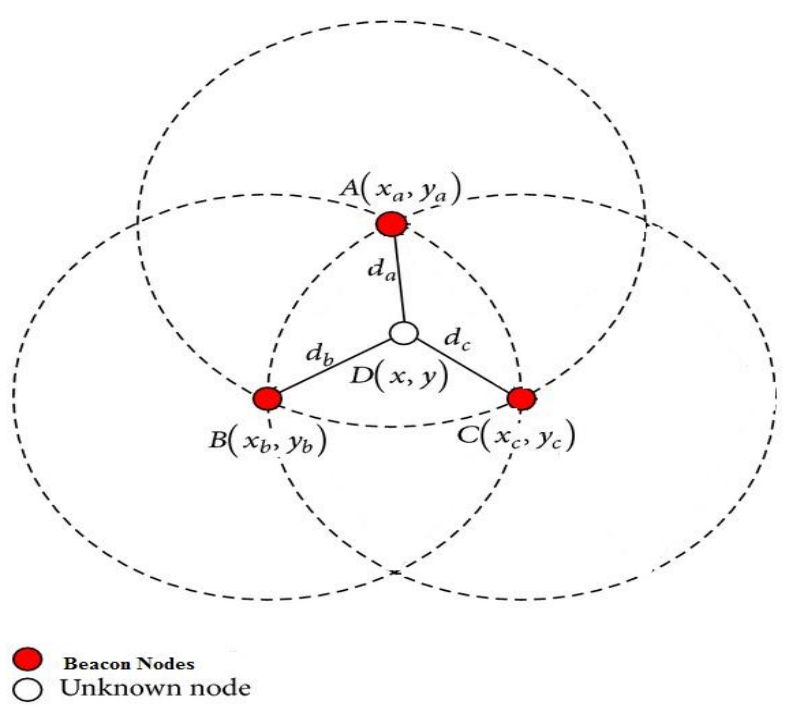

Fig 4: Trilateration Algorithm

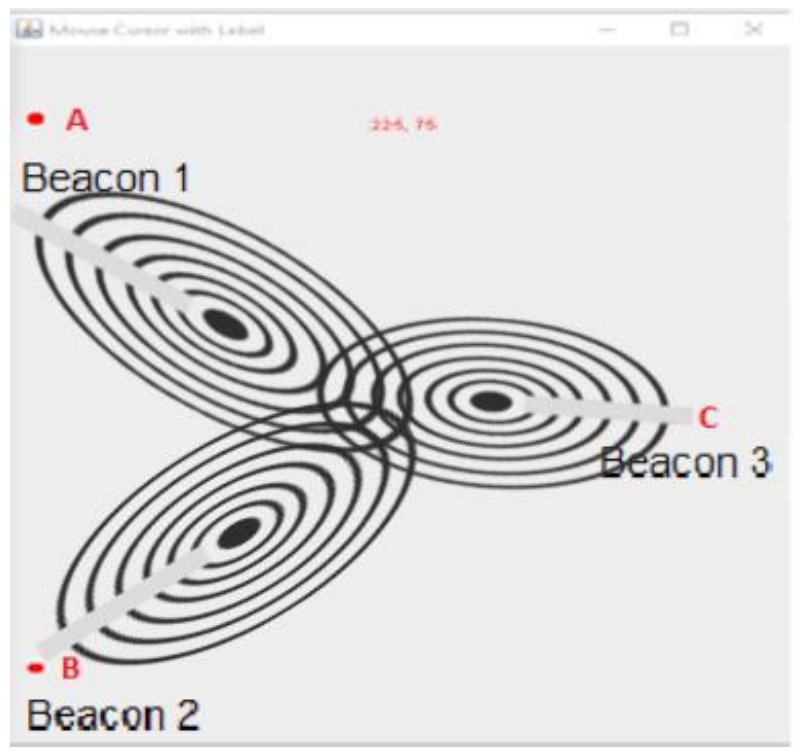

Fig 5: Beacons and user location map in Java 2D

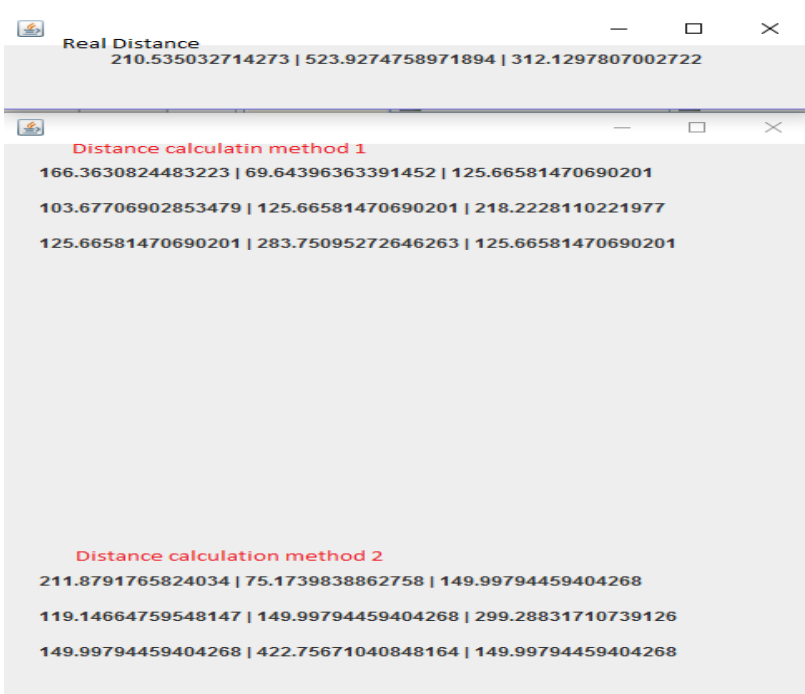

Fig 6: Distance in Java 2D
As shown in the Fig. 5 and Fig. 6 to analyze the capabilities of distance conversion functions we simulate the real environment in Java2D and compare real distance with a distance using RSSI. Observing distance error from one beacon determined error rate will be high when it comes from three beacons.

Thus, in order to get the $(\mathrm{x}, \mathrm{y})$ coordination fingerprinting method based on the suggested methodology.

Table 1. Fingerprint database structure

\begin{tabular}{|c|c|}
\hline Fingerprint & Location \\
\hline $\mathrm{FP} 1=\left\{R S S I_{1}^{F P 1}, R S S I_{2}^{F P 1}, \ldots, R S S I_{m}^{F P 1}\right\}$ & $\left(x_{1}, y_{1}\right)$ \\
\hline $\mathrm{FP} 2=\left\{R S S I_{1}^{F P 2}, R S S I_{2}^{F P 2}, \ldots, R S S I_{m}^{F P 2}\right\}$ & $\left(x_{2}, y_{2}\right)$ \\
\hline$\ldots \ldots \ldots \ldots \ldots \ldots \ldots \ldots \ldots \ldots \ldots \ldots \ldots \ldots$ & $\ldots \ldots$ \\
\hline $\mathrm{FPN}=\left\{R S S I_{1}^{F P N}, R S S I_{2}^{F P N}, \ldots, R S S I_{m}^{F P N}\right\}$ & $\left(x_{N}, y_{N}\right)$ \\
\hline
\end{tabular}

Then location fingerprints (Filtered RSSI) are gathered up by dividing the place into rectangular grids and stored in the database. Finally, this will be used to navigate the mobile node.

\subsection{Location Coordination Output}

The location coordination output (Fig.8) clearly indicates the course of the moving target. By using the fingerprint database structure is given in TABLE $\mathrm{I}$, the object location is calculated.

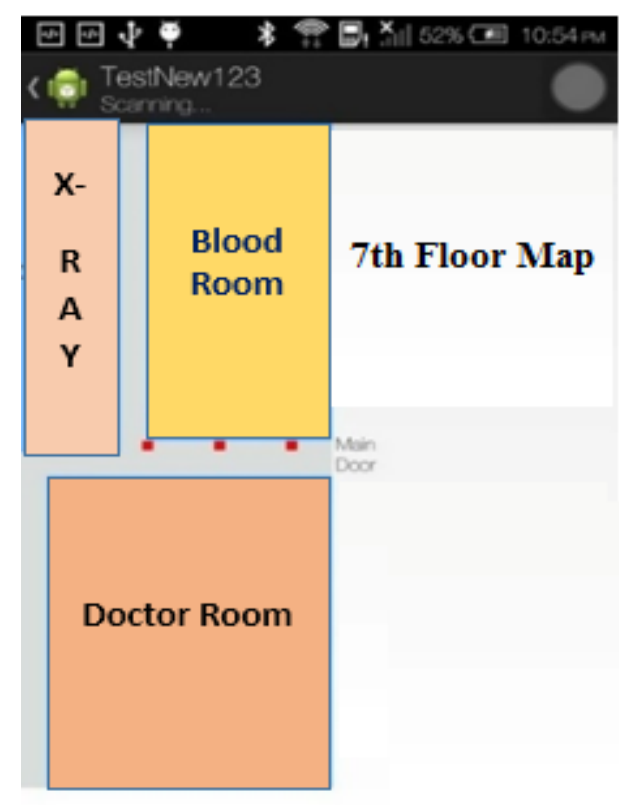

\section{$\supset \quad 0 \quad \square$}

Fig 7: Location coordination output

\section{RESULTS AND DISCUSSION}

This Section discusses the experimental setup that followed in establishing a relationship between RSSI values and distance. 
This purpose the Sri Lanka Institute Of Information Technology $7^{\text {th }}$ floor was used as a sample test environment. The idea is to make a relationship to determine the error and to check which filter is most suitable for smoothing the RSSI values.

Fig. 8 shows the row RSSI and RSSI after performing feedback filtering which remove outliers. Red line shows very high RSSI and low RSSI are deducted.

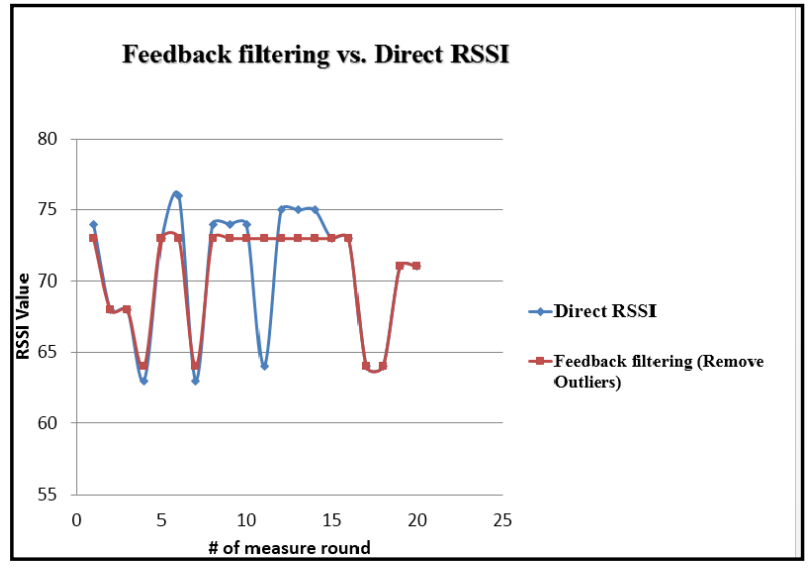

Fig 8: Feedback filtering vs. direct RSSI

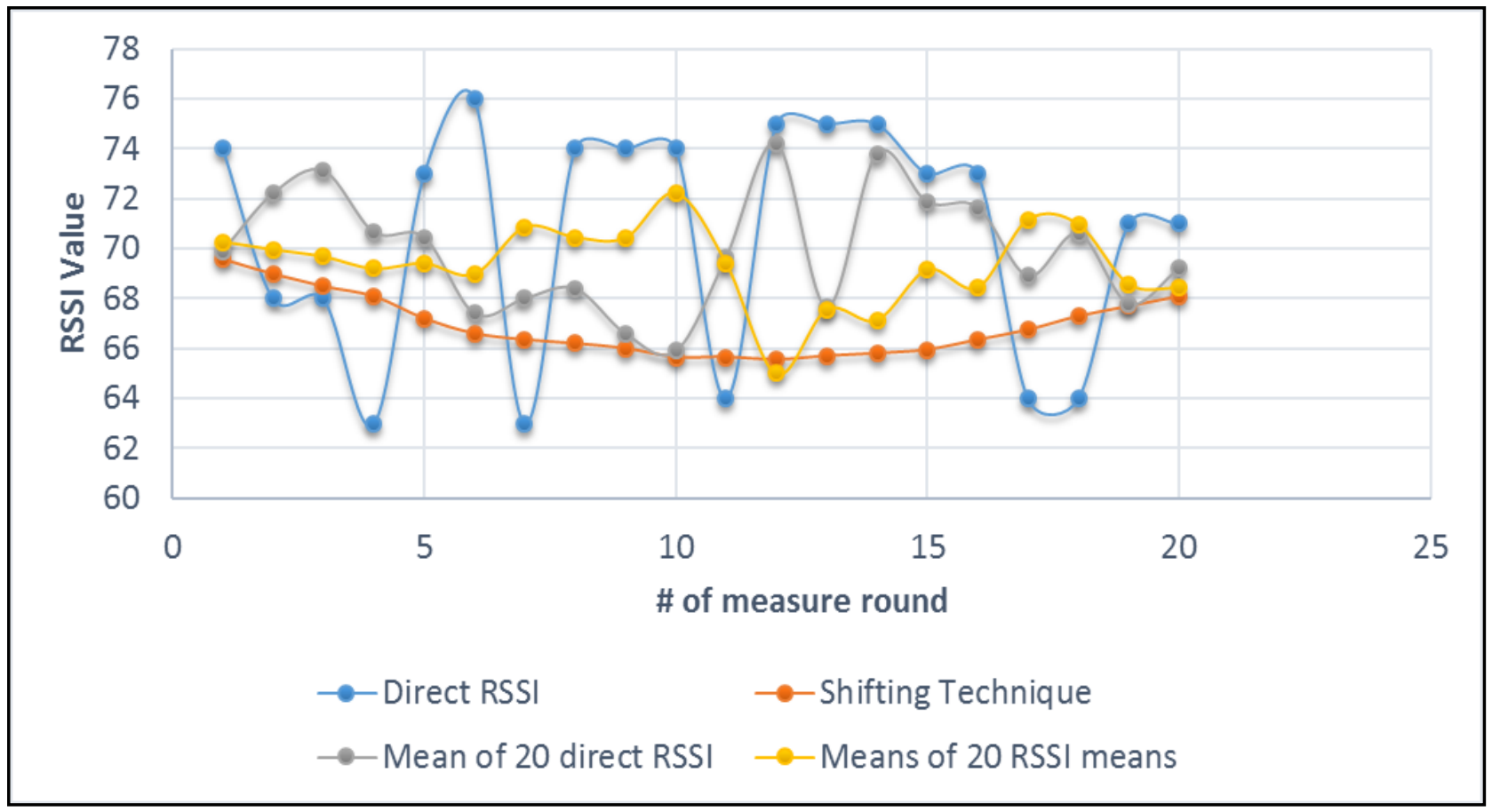

Fig 9: Comparison of filtering methods

The authors followed three methods to find the static RSSI values.

- Get the RSSI mean value from 20 RSSI values.

- Get the RSSI mean value from 20 RSSI mean values.

- Get the RSSI mean value by shifting RSSI values.

In order to smooth the RSSI value three filtering techniques were tested in par with methods mentioned above. Fig. 9 presents the experimental comparison of some filtering techniques. It is obvious to the common eye that new shifting technique, smooth and perform the best out of all.

Comparison with distance and is verified after choosing the shifting technique.

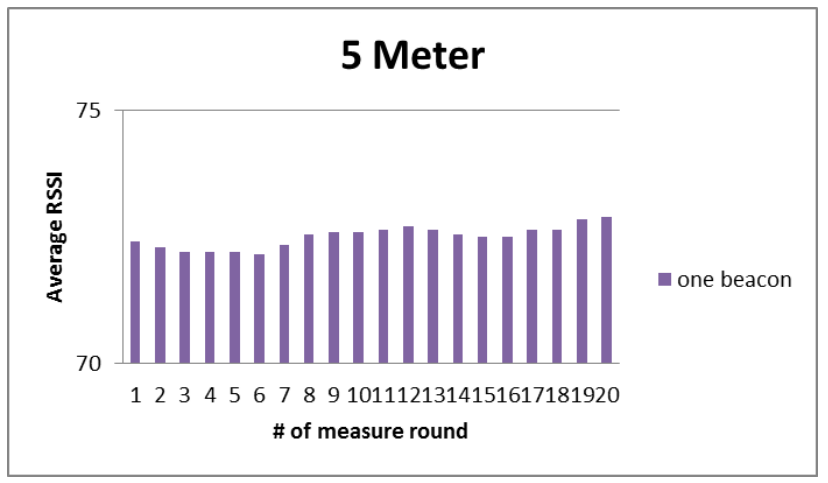

Fig 12: RSSI from $5 m$ distance 


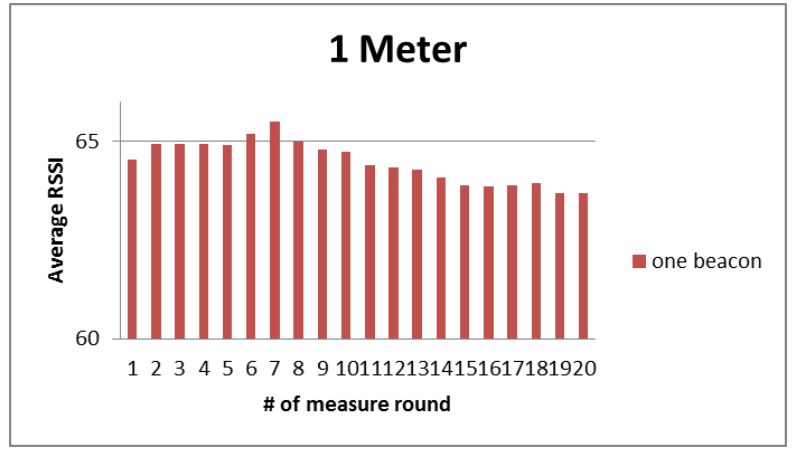

Fig 10: RSSI from 1m distance

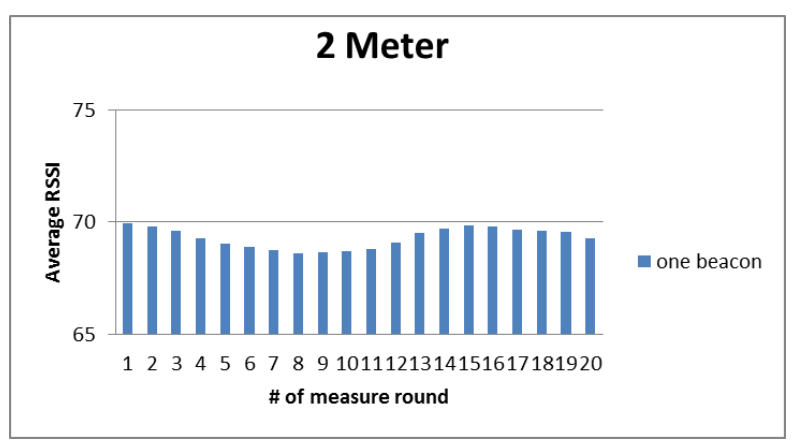

Fig 11: RSSI from 2m distance

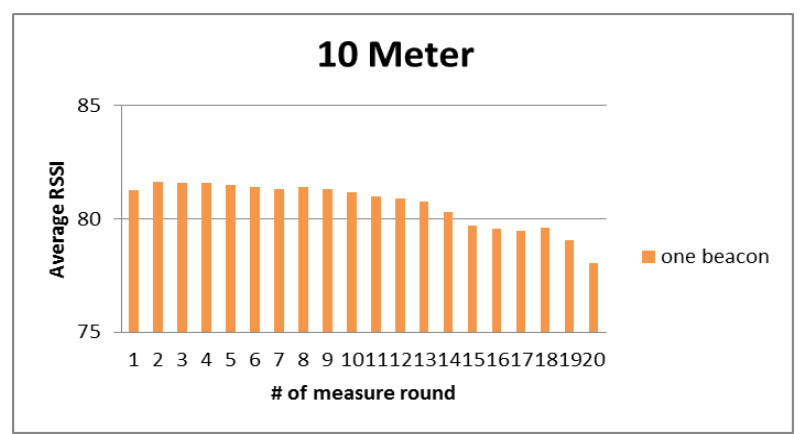

Fig 13: RSSI from 10m distance

Fig. 15 shows before executing shifting technique RSSI values are overlapping with different length. Therefore, it is very hard to define an exact RSSI range to the particular grid. Hence to overcome a challenge mentioned above, the shifting algorithm is introduced. Fig. 10- 14 shows RSSI value range is split up from one length to another.

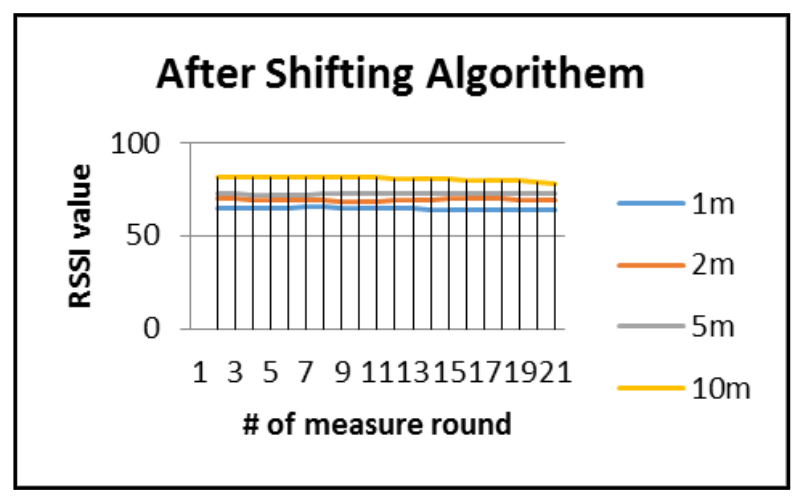

Fig 14: After shifting algorithm

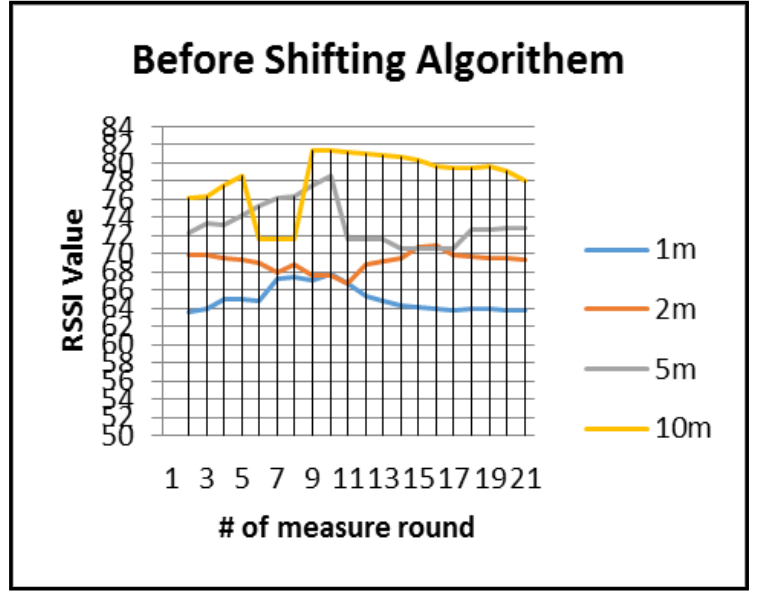

Fig 15: Before shifting algorithm

\section{CONCLUSION AND FUTURE WORKS}

This research work demonstrated that the novel approach to finding indoor localization by omitting errors in existing algorithms.

In this research project, it is clear now that indoor positioning is a difficult task. Topology constraints in indoor environments are much more complex than those in outdoor environments. In this research, authors built a smartphone application to allow us to make measurements of BLE signals as well as implementing ways to improve them. We took a series of tests in a numeral of different distances to identify how the signals are impressed. Authors looked into the possible ways to improve the data as it is not suitable as raw information. In the end, came up with a novel technique (shifting) to obtain the static RSSI values to navigate the user accurately. The experimental results proved the proposed mathematical method can shorten the average error, and it is invariably safer than the other existing interference avoidance algorithms. This research can use for reference for future software developers in the field of indoor location systems and Bluetooth proximity zones that would make use of Smartphones.

In the future work planned to implement Trilateration together with fingerprinting online phase and learning benefits from the role of shifting filter in the measurement stage to minimize the errors.

\section{ACKNOWLEDGMENT}

This work has supported by the Sri Lanka Institute of the Information Technology, Malabe Sri Lanka.

\section{REFERENCES}

[1] Dr. Rainer Mautz, Indoor Positioning Technologies. Institute of Geodesy and Photogrammetry, Department of Civil, Environmental and Geomantic Engineering, ETH Zurich, February 2012.

[2] Manh Hung V. Le, Indoor Navigation System for Handheld Devices. Worcester Polytechnic Institute Worcester, Massachusetts, USA, October 2009.

[3] Zhen Fang, Zhan Zhao, Dao Geng, Yundong Xuan, Lidong Du,Xun Xue Cui, "RSSI Variability Characterization and Calibration Method in Wireless Sensor Network", International Conference on 
Information and Automation, June 20-23, 2010, pp.15321537.

[4] A. Awad, T. Frunzke, and F. Dressler, "Adaptive distance estimation and localization in won using RSSI measures," in DSD, 2007, pp.471-478.

[5] H. Y. Shi, "A new weighted centroid localization algorithm based on RSSI," in Proc. International Conference on Information andAutomation, 2012, pp. 137-141.

[6] Pei-gang SUN, Hai ZHAO, Ding-ding LUO, "Research onRSSI-based Location in Smart Space," ACTA ELECTRONICASINICA, 2007, 35(7), pp:1240- 1245.

[7] Diaz, J. M.; Maues, Rodrigo de A.; Soares, Rodrigo B.;Nakamura, Eduardo F., Figueiredo, Carlos M. S."Bluepass:An indoor Bluetooth-based localization system for mobile applications, "Computers and Communications (ISCC), 2010 IEEE Symposium on , vol., no., pp.778-783, 22-25 June 2010 doi: 10.1109/ISCC.2010.5546506.

[8] Yim J., Jeong S., Gwon K. and Joo J. (2010) Improvement of Kalman filters for WLAN based indoor tracking. ExpertSystems with Applications, 37(1), pp.426-433.

[9] Kotanen A., Hännikäinen M., Leppäkoski H. and Hämäläinen T.D., "Experiments on local positioning with Bluetooth," International Conference on Information Technology Computers and Communications, pp.297, 2003. DOIs 10.1109/ITCC.2003.1197544.
[10] Mao G., Baris, Fidan and Anderson D.O., "Wireless sensor network localization techniques," The International Journal of Computer and Telecommunications Networking.

[11] Avanti K., "Comparative study of RSS-based collaborative localization methods in wireless sensor networks," MSc Thesis, Toulouse School of Graduate Studies, 2006.

[12] Roxie A., Gaber J., Wack M., and Nait-S.M.A., "Survey of wireless relocation techniques," IEEE Workshop on Service Discovery and Composition in Ubiquitous and Pervasive Environments (SUPE), 2007. DOIs, 10.1109/GLOCOMW.2007.4437809.

[13] Zhou S. and Pollard J.K., "Position measurement using Bluetooth," IEEE Transactions on Consumer Electronics, vol.52, no.2, p.555, 2006. DOIs, 10.1109/TCE.2006.1649679.

[14] O.Oguejiofor, A.Andiedu, H.Ejiofor, and A.Okolibe,(2013)" Trilateration Based Localization Algorithm for Wireless Sensor Network" IJISME.ISSN: 2319-6386, Volume-1, Issue10.Availabe:http://www.http://ijisme.org/attachments/Fil e/v1i10/J04470911013.pdf.

[15] L. Pei et al., "Inquiry-based Bluetooth Indoor Positioning via RSSI Probability Distributions", Second International Conference on Advances in Satellite and Space Communications, 2010, pp. 151-156. 